\title{
COVID 19 Data Visualization through Automatic Phase Detection
}

Pradipta Biswas, KamalPreet Singh Saluja, Somnath Arjun, LRD Murthy, Gowdham Prabhakar,

Vinay Krishna Sharma, Jeevitha Shree DV

Indian Institute of Science

\begin{abstract}
This paper presents a new interactive visualization system to represent and compare rate of spread of COVID 19 pandemic across different countries over time. We surveyed existing visualization techniques used in various websites and media outlets and introduced use of a knee detection algorithm that divides the exponential spread in multiple linear components. A set of use cases demonstrates the utility of the system in comparing the spread across different countries for both analysis and prediction.
\end{abstract}

\section{Introduction}

Data visualization or information visualization always played a key role in scientific analysis. Starting from John Snow's analysis of cholera epidemic in 1854, till now a good visualization can represent inherent trend in data, which may not otherwise be visible from raw numbers. The recent COVID 19 pandemic poses new challenges to data scientists too for its vast and rapid spread and significant economic impact. This paper investigates existing visualization techniques used to represent ongoing spread of the pandemic and then proposes a new web based interactive visualization tool (http://cambum.net/Covid19/index.html). In particular, we have developed and deployed a web based interactive software that indicates spread of COVID 19 pandemic by automatically dividing the duration of spread based on rate of increase of cases. It uses a Knee detection algorithm to progressively divide the duration. The shape of the graphs (like linear, parabolic or exponential) can be compared at different stages and countries with respect to the average number of new cases and deaths. Instead of dividing the timeline uniformly for all countries on ad hoc basis, the website automatically detects phase of transmission in a data centric manner for each country. Our analysis and website can be used to

Undertake comparative analysis among countries

Automatically detect and compare phases of spread across different countries

Prevent spread by taking examples from other countries

In the following paragraphs, we listed the existing visualization techniques and their short comings and then presented our proposed approach with case studies of representative use cases. 


\section{Existing Visualization Techniques}

A plethora of work has been done on visualizing COVID-19 data since the outbreak of the pandemic. Those visualizations can be primarily classified into two groups visualization with zero or less interactivity represents the first group and complex interactive visualization techniques and tools represents the second. Static visualization [1,2] uses basic graphs such as bar and line graphs for representing attributes of COVID-19 dataset. Washington Post [1] used collection of bar graphs for comparing new cases and deaths between countries. Each chart represented a country and each bar of every chart displays the number of cases or deaths. Financial times developed a visualization [2] with series of line charts for comparing daily deaths. Bar and line charts were combined together to form composite static graph [3] for showing two different data attributes - bar chart was used to display number of new cases with line charts showing the change of percentage of daily deaths. The chart was annotated with events like schools closed, complete lockdown started and so on. A few visualizations allowed user interaction $[4,5]$ like hovering the mouse over bars and points in line charts. Worldometers website was one such example which used two graphs for showing total number of cases and new cases every day. These type of representation is quite easy to implement and understand but it can miss relevant information. Two other visualizations [6] used more interactivity than the previous example with line charts to represent second derivative of daily number of cases and deaths displaying their progression rate. Majority of websites $[1,8,9,10,11]$ used interactive world map charts or world map globe as their primary layout for the visualization. Almost all these visualizations had hover and click interactivity displaying data attributes like number of cases, number of deaths, active cases, recovered cases, number of fatal cases and so on. Flourish studio [13] used four types of visualization techniques for managing distinct information with live updating graphics as follows

1. Interactive trajectory chart for number of cases and deaths

2. Sunburst was used for breaking the impact of virus by countries

3. Searchable line chart for number of confirmed cases and recovered cases

4. World map charts for number of cases and deaths

Wikipedia and Tableau Public $[12,15]$ used interactive stacked bar charts for showing attributes like active cases, total cases, deaths, recoveries and number of people hospitalized. Interactive trajectory chart was used in International SOS website [14] to compare rates of new cases each day to determine how fast the outbreak is growing in countries. Users can toggle between logarithmic axes (default) and linear axes. This tool consists of two more charts, first one compares between the number of cases against the number of tests performed, second one shows the cumulative tests carried out per 1000 people in countries. 
In summary, we have identified following challenges while visualizing data related to the spread of the COVID 19 pandemic

1. Range of data: The number of cases and deaths range from a tenth of million to less than hundred in different states, regions and countries. It is difficult to plot this wide range of values sharing a single scale. The trend may look flat even if it is not in countries or states where the number of new cases is in hundreds when plotted with a country or region where it is in thousands.

2. Data transformation: Usually in science, researchers use log or semilog transformation for plotting data with a wide range. However, presently the COVID statistics has a huge influence on people's perception of threat and a flat logarithmic curve may undermine the effect of the disease among common people if they do not follow the actual data transformation.

3. Proportional statistics: A plethora of statistics is defining new variables like number of people affected per capita or per million population or proportion of death with respect to total number of affected people. These statistics often exaggerate the problem for both small and large countries. For example, a huge number of death may seem a small proportion of per million people in a country like India or China and only a few deaths may seem a big number for a small and less populous country.

4. Information overload: When the graphs present data for multiple states or countries, all graphs turn indistinguishable at the early stage of the pandemic and countries where the spread has not yet reached peak clutter together even for a set of line graphs.

5. Difficulty in trend analysis: The WHO and Bing website undertook a novel approach of relating the total number of cases with radius of a semi-transparent circle and placing it at geographic location. While this visualization can present a snapshot of present status but do not provide an indication to trend of spread over time. The traditional trend analysis graphs are often not interactive and show a similar trend for many countries in a cluttered way.

\section{Proposed Approach}

We have proposed the following approach in visualizing the spread of the pandemic for comparison and prediction among different states and countries

1. Automatic phase detection: At present, the spread of COVID-19 is shown to have an exponential trend in most countries. An exponential curve is difficult for both comparison and prediction as it has a vertical asymptotic trend to infinity. 
Our analysis automatically divides the duration of spread of the disease based on rate of increase in new cases using a knee detection algorithm [16, 17] and shows a set of three graphs which are easier to interpret and extrapolate than a single exponential graph. The shape of the graphs (like linear, parabolic or exponential) can be compared at different stages and countries with respect to the average number of new cases and deaths. We used two different $Y$ axes to indicate number of cases and deaths.

2. Combination of bar and line graphs: A line graph is good to indicate trend and takes least screen estate. However, if it is close to any axes, then the line often overlaps with the axis itself and may seem discontinuous. However, a column graph uses more screen estate and thus more visible than a line graph while depicting a parameter with small values. When we plot both number of total number of cases and number of new cases per day using same $Y$ axis, we used a line graph for total number of cases and a column graph for number of new cases per day. Same logic is also followed for showing number of deaths.

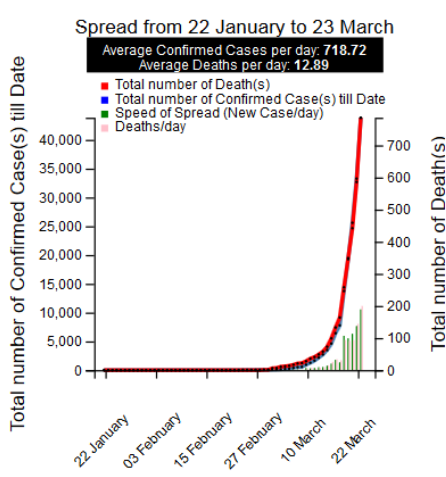

Date - US

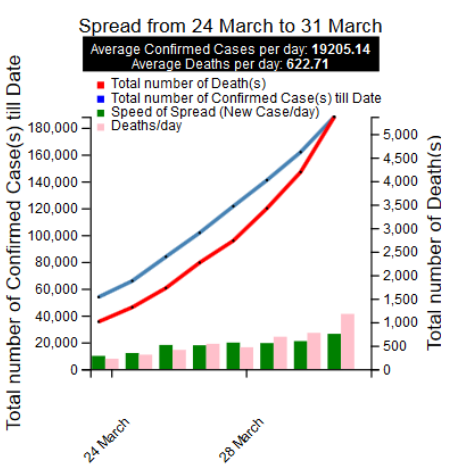

Date - US

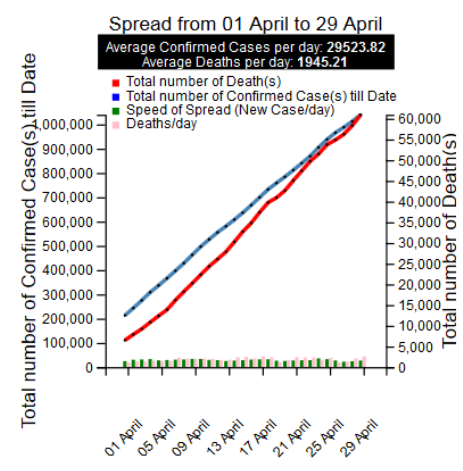

Date - US

Figure 1. Set of three graphs combining line and bar charts

3. Combination of Line graph and Pie chart: While three graphs are used for comparing different phases of spread for a single country or state, we have used a combination of line graph and pie chart (figure 2) to compare the spread among multiple countries or states and their daily contributions to number of new cases and deaths. The line chart only compares the recent past among different countries or states. The pie chart shows the ratio of number of cases and deaths for a particular date of different countries or states with respect to the world or India respectively. 

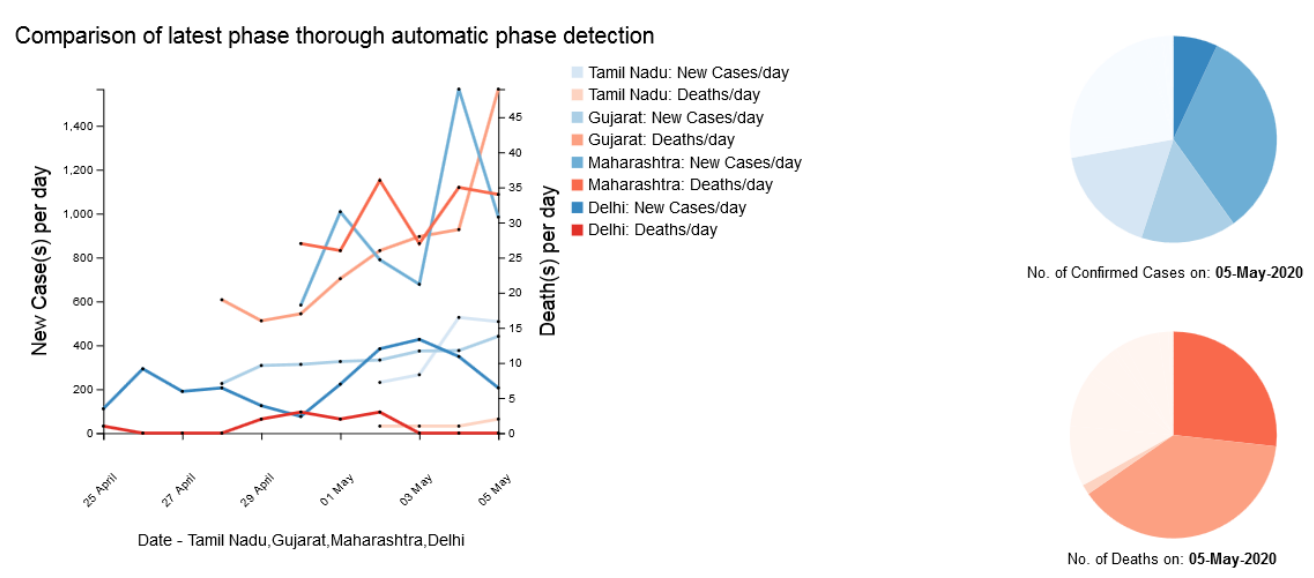

Figure 2. Comparative analysis using line graph and pie chart

4. Comparative statistics on daily basis: For individual state or country, we divide the total duration in three separate graphs. Each of these three graphs (figure 1) show total number of cases and deaths as well as number of new cases and deaths per day. However, for comparison among multiple regions (figure 2), we use only number of new cases and deaths per day for following reasons

a. Number of new cases and deaths each day have a smaller range than total number of cases

b. Number of new cases and deaths per day depict the speed of spread and indicates the trend better than total number of cases

\section{Use Cases}

The website generated visualization to easily compare the speed of spread. Figure 3 below shows a comparison of number of new cases and deaths per day for four countries having total number of cases between 13500 and 14500 on $18^{\text {th }}$ April 2020. It may be observed that

Only Austria has a decreasing trend in terms of new cases per day

Number of deaths are increasing at Ireland

According to our analysis, the spread is lagging by a couple of days in India, 5 days in Peru and a week in Ireland than Austria 
Comparison of latest phase thorough automatic phase detection

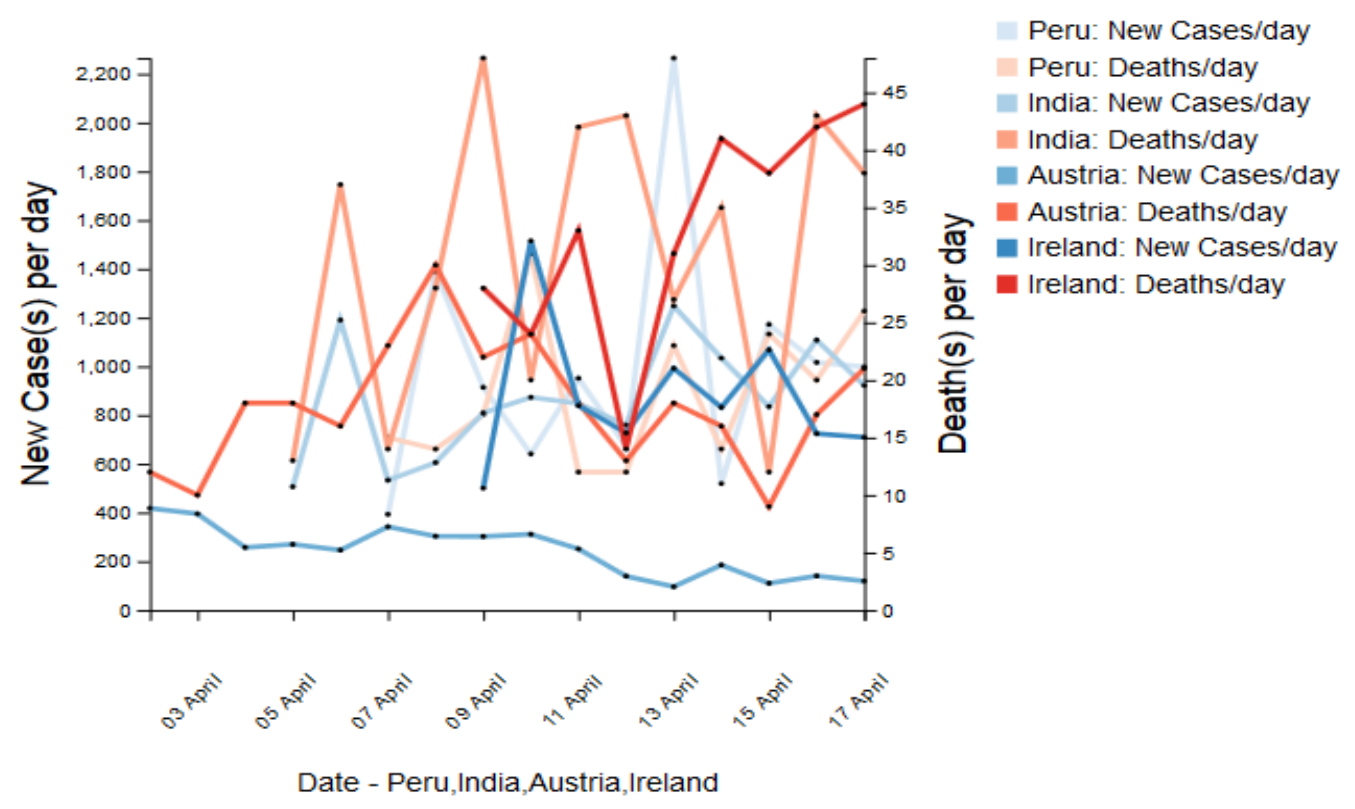

Figure 3. Comparison graph

By comparing average number of new cases per day at different phases of trend, we can estimate the rate of spread in upcoming future. For example, as of $28^{\text {th }}$ April 2020, the average number of new cases per day in Karnataka (14.22) was similar to the same parameter at Bolivia (15.68) from $25^{\text {th }}$ March to $13^{\text {th }}$ April and at Jordan (15.33) from $14^{\text {th }}$ March to $4^{\text {th }}$ April. However, the spread took different directions in Bolivia and Jordan, comparing the situations there in mid April may be useful for Karnataka at present time.

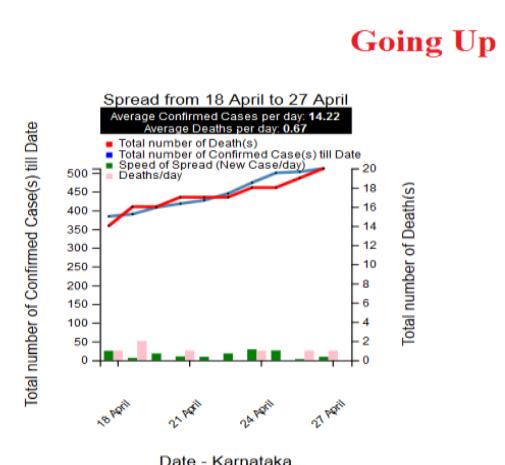

Going Down

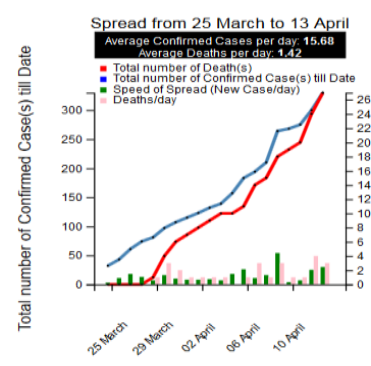

Date - Bolivia

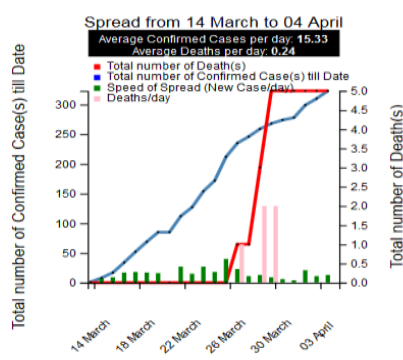

Date - Jordan

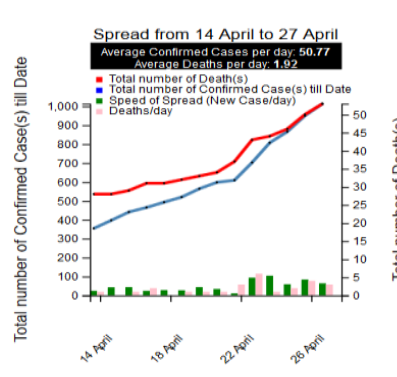

Date - Bolivia

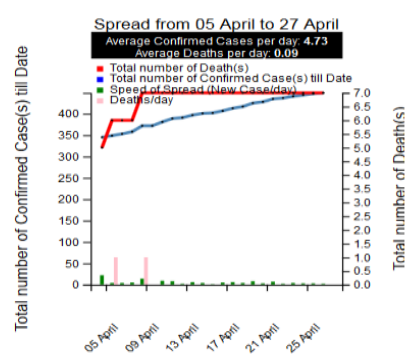

Date - Jordan

Figure 4. Similarity in trend analysis 
Our website shows comparisons of recent past with present state of COVID 19 spread in multiple countries. For example, the following graphs generated on $14^{\text {th }}$ May show that till mid-April, number of new cases was higher in Germany than UK although at present time the trend is reversed as the pandemic is better contained in Germany than UK (figure 5).

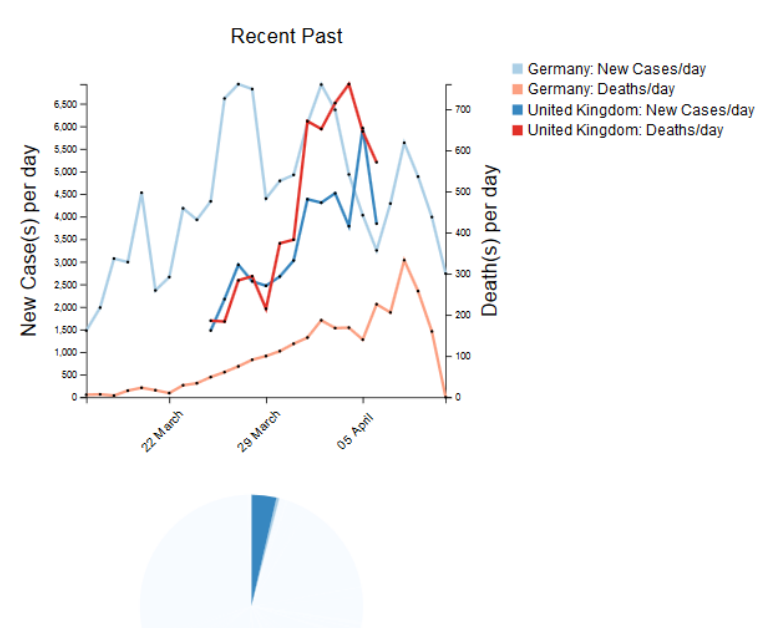

No. of Confirmed Cases on: 14-May-2620

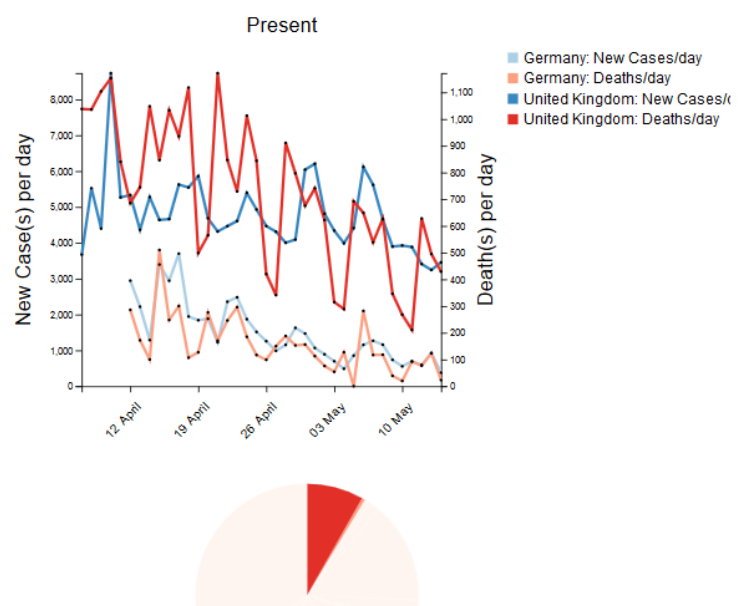

No. of Deaths on: 14-May-2020

Figure 5. Comparative analyses at different phases between UK and Germany

A similar analysis was undertaken on India and European countries on $21^{\text {st }}$ May (figure 6). It may be seen that India had much lower number of cases earlier but now the number of cases are higher than European countries and it is increasing. However, number of deaths is lower, it may be due to the lag in spread by which time medical facilities got more time to prepare.

We undertook an exhaustive comparative analysis of all countries and states on $29^{\text {th }}$ April and generated the following graph (figure 7). The following graphs represent a comparison of average number of new cases per day across different countries and Indian states in recent past and present. The graphs should be magnified and read in clockwise direction from top which will indicate increasing trend. The green line and labels represent present while the red line and orange labels represent recent past. The $Y$-axis represent number of new cases per day. 
Comparison of COVID 19 spread in India with European Countries on 21st May 2020
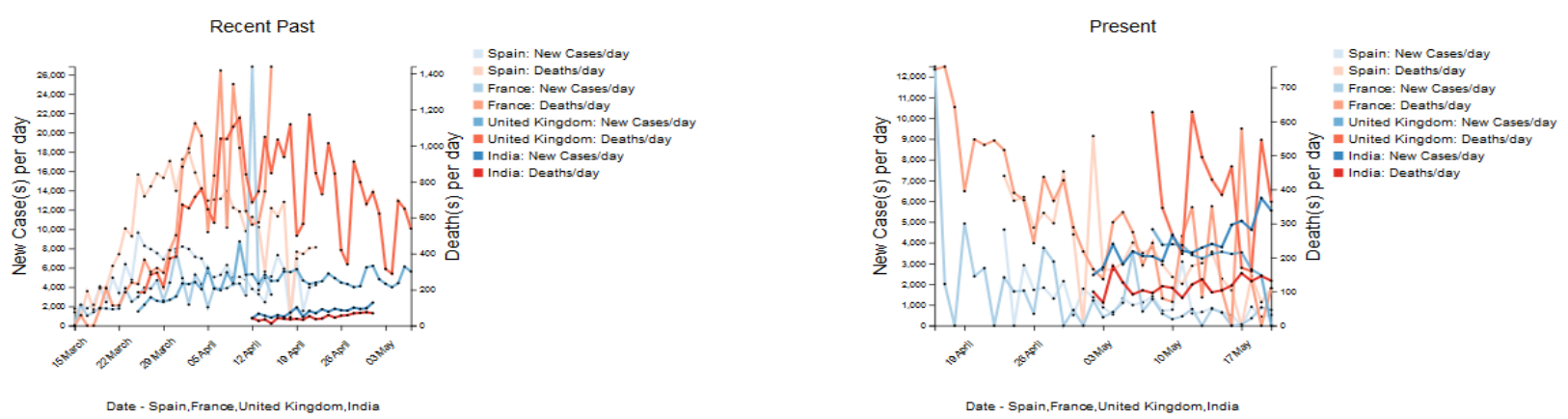

Figure 6. Comparative analyses at different phases between India and European countries

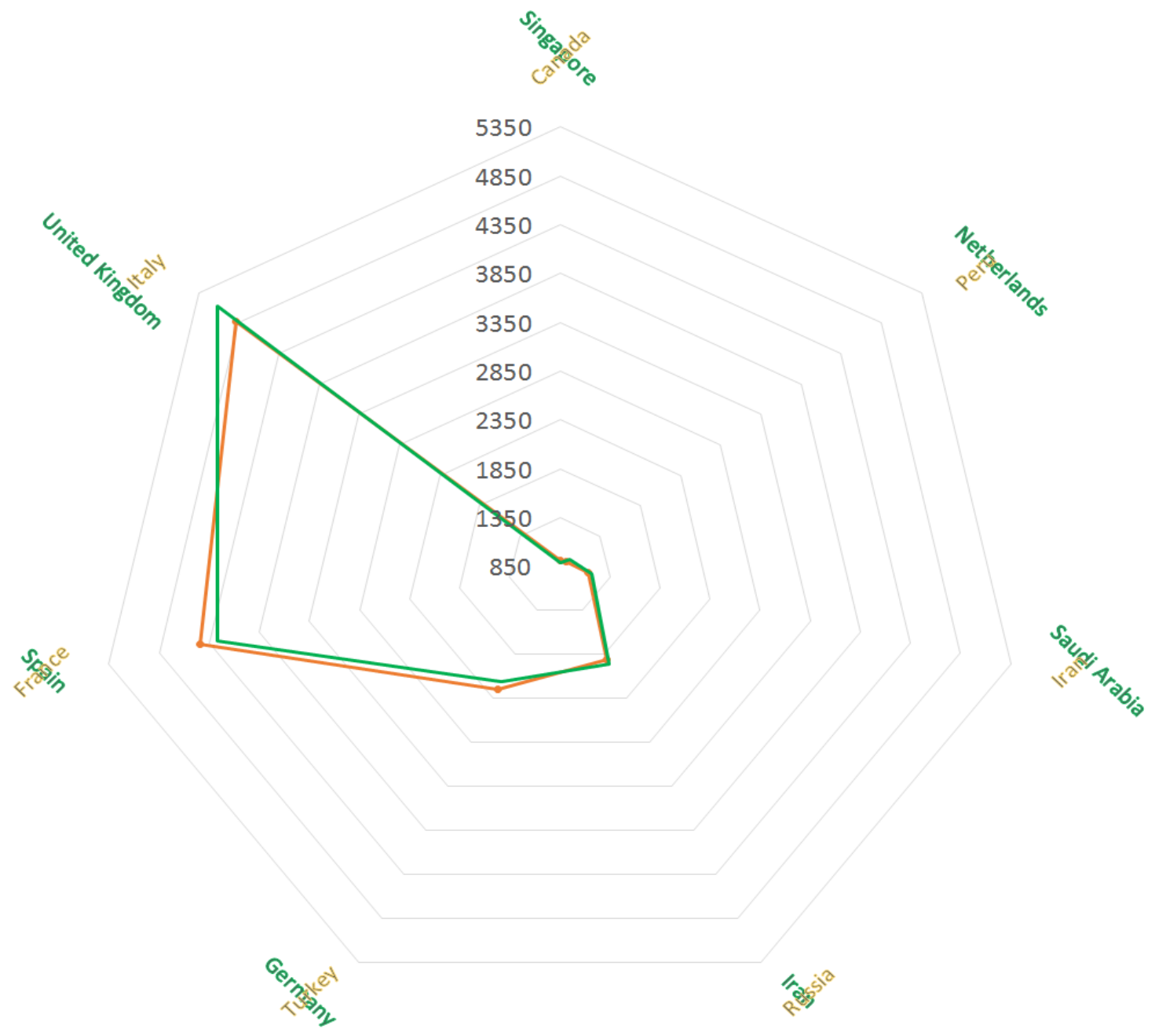

Figure 7. Radar charts comparing rate of spread comparing countries where number of new cases per day is greater than 850 on $29^{\text {th }}$ April 


\section{Interaction with Alternative Modality}

In order to make the interaction with our dashboard convenient and inclusive, we integrated Automatic Speech Recognition (ASR) and Text-to-Speech (TTS) features into our web application. We used webspeech API [7] to implement these features. We included navigation features like scroll up and scroll down along with the focus on selecting various countries through voice commands. Once we obtain the access to user's microphone, we classify the voice commands into three categories: a country name or a navigation command or an unrecognizable text.

We have set the ASR recognition confidence threshold as $70 \%$ and any predicted text with a lower confidence score would be rejected. On recognizing a country's name, we render the information on COVID-19 cases related to that country and use TTS engine to read out the necessary information. This includes the total number of infections, number of recovered cases and number of deaths. This also includes the information on new cases per day and new deaths per day across all 3 phases detected by our knee detection algorithm. On recognizing a navigation voice command, we simulate scroll events on our web application. On recognizing a command that is unrecognized, we use Levenshtein distance to suggest a country name, based on the difference between our dictionary and predicted text. The string in our dictionary with least Levenshtein distance against the predicted text would be suggested to the user. A video demonstration can be found at https://youtu.be/lyvwK9enFtw

\section{Conclusions}

This paper summarizes existing visualization techniques used to visually represent spread of COVID 19 pandemic across different countries and presents a new interactive web-based system that automatically divides the duration of spread into three phases. A set of use cases demonstrates the utility of the new system in terms of comparing rate spread across different countries and different times. The system can also indicate rate and trend of spread over time and by comparing with past examples, the system can also be used to predict future rate of spread. The system is also integrated to automatic speech recognition and text to speech features to disseminate information to people with different range of abilities.

\section{References}

1) Mapping the Worldwide Spread of the Coronavirus. The Washington Post, WP Company, 24 Apr. 2020, www.washingtonpost.com/graphics/2020/world/mapping-spread-new-coronavirus/.

2) Burn-Murdoch, John, et al. Coronavirus Tracked: the Latest Figures as the Pandemic Spreads: Free to Read. Financial Times, Financial Times, 25 Apr. 2020, www.ft.com/coronavirus-latest. 
3) New Zealand flattening the curve, 27/04/2020, https://www.dailymail.co.uk/news/article$8259269 /$ Australia-outperforming-New-Zealand-controlling-coronavirus-curve-without-stagefour-lockdown.html

4) Coronavirus Cases: Worldometer, www.worldometers.info/coronavirus/

5) 91-DIVOC. Flip the Script on COVID-19, 91-divoc.com/pages/covid-visualization/.

6) Rémy, D. What's Wrong with COVID-19 Data Visualizations, and How to Fix It. Medium, Towards Data Science, 5 Apr. 2020, towardsdatascience.com/whats-wrong-with-covid-19-datavisualizations-and-how-to-fix-it-3cdc9adc774d.

7) Webspeech API, https://developer.mozilla.org/en-US/docs/Web/API/Web Speech API

8) World Health Organization, World Health Organization, covid19.who.int/.

9) Mamoon, N, and Rasskin, G. COVID-19 Visualizer. Visualizer, www.covidvisualizer.com/

10) Bing, Microsoft, bing.com/covid/local/india.

11) COVID-19 - Analysis, Visualization \& Comparisons. Kaggle, Kaggle, 7 Apr. 2020, www.kaggle.com/imdevskp/covid-19-analysis-visualization-comparisons.

12) Tableau Public, public.tableau.com/profile/oregon.health.authority.covid.19\#!/vizhome/ OregonHealthAuthorityCOVID-19DataDashboard/COVID-19EPIConfirmed.

13) COVID-19: Free Live Mobile-Friendly Visualizations for Use on Any Website. Flourish, flourish.studio/covid/.

14) Interactive Covid-19 Data By Location. Footer Image, pandemic.internationalsos.com/2019ncov/covid-19-data-visualisation.

15) 2020 Coronavirus Pandemic in India. Wikipedia, Wikimedia Foundation, 28 Apr. 2020, en.wikipedia.org/wiki/2020_coronavirus_pandemic_in_India.

16) Satopaa, V., Albrecht, J., Irwin, D., \& Raghavan, B. (2011). Finding a 'kneedle'in a haystack: Detecting knee points in system behavior. 166-171. In 31-st International Conference on Distributed Computing Systems.

17) Saluja KPS, Jeevithashree Dv, Arjun S. and Biswas P., Analyzing Eye Gaze Movement of Users with Different Reading Abilities due to Learning Disability, 3rd International Conference on Graphics and Signal Processing (ICGSP 2019) 